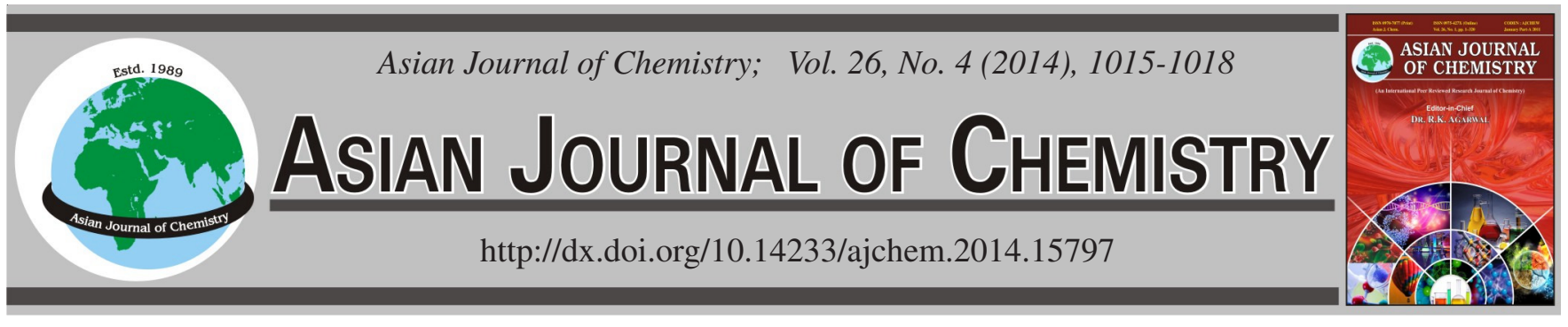

\title{
Pd/C-Catalyzed Ligand-Free Hiyama Cross-Coupling Reaction of Aryl Halides Under Aqueous Conditions
}

\author{
Nagaraju Mittapelly ${ }^{1,2, *}$, Khagga Mukkanti ${ }^{2}$ and Buchi Reddy Reguri ${ }^{3}$
}

${ }^{1}$ Inogent Laboratories Private Limited, A GVK BIO Company, 28A, IDA, Nacharam, Hyderabad-500 076, India

${ }^{2}$ Centre for Chemical Sciences and Technology, I.S.T. Jawaharlal Nehru Technological University, Kukatpally, Hyderabad-500 085, India

${ }^{3}$ Orchid Chemicals and Pharmaceuticals Ltd., Sholinganallur, Chennai-600 119, India

*Corresponding author: E-mail: nagunitrogen@yahoo.co.in

Received: 18 May 2013;

Accepted: 7 August 2013;

Published online: 15 February 2014;

AJC-14690

\begin{abstract}
A mild and efficient ligand-free Hiyama cross-coupling reaction using $\mathrm{Pd} / \mathrm{C}$ as a catalyst under aqueous conditions has been developed. A wide variety of aryl bromides undergo the cross-coupling with aryl siloxanes in excellent yields without the use of any additives. The operational simplicity and the mild reaction conditions add to the value of this method as a practical alternative to the Hiyama crosscoupling of aryl and heteroaryl halides.
\end{abstract}

Keywords: Hiyama cross-coupling, Aryl halide, Pd/C.

\section{INTRODUCTION}

The efficient formation of carbon-carbon bonds is among the most crucial transformations in synthetic chemistry. The palladium-catalyzed aryl-aryl cross-coupling reaction is one such route to these bond formations and the formed biaryl substructure is a widely occurring component of biologically active and functional molecules ${ }^{1}$, which includes the pharmaceuticals ${ }^{2}$ valsartan, telmisartan, felbinac, losartan, imatinib, the agrochemical Boscalid ${ }^{3}$, the natural product ${ }^{4}$, biphenomycin and liquid crystals for LCD screens ${ }^{5}$.

Traditional methods that have been employed to accomplish this biaryls include Pd-catalyzed Suzuki ${ }^{6}$, Negishi ${ }^{7}$, Kumada $^{8}$, Hiyama ${ }^{9}$ and Stille ${ }^{10}$ reactions. Recently, Hiyama cross-coupling reaction has gained increased attention as organosilicon reagents are commercially available at relatively low cost or can be easily prepared. These reagents are also nontoxic and quite stable to the presence of other functionalities and to a variety of reaction conditions.

Generally, phosphine ligands are used to complex and activate the palladium species and excellent results have been reported for the palladium-catalyzed Hiyama cross-coupling reaction in presence of fluoride anion ${ }^{11}$. However, phosphine ligands are undesirable because of their toxicity and air as well as moisture sensitive with conversion to, e.g., phosphine oxide species and the requirement of corrosive fluoride anion for the activation of organosilanes ${ }^{6,9,10}$. The potential of the aqueous Hiyama reaction was recognized with the development of sodium hydroxide as an efficient promoter and several examples of the Hiyama reaction in aqueous medium using nitrogen based ligands were recently demonstrated ${ }^{12,13}$.

However, the main drawback of homogeneous catalysis is associated with separation and reuse of the catalyst. This condition leads to a loss of expensive metal (and ligands) and to the presence of metal impurities in the products. The complete removal of residual metals is a huge problem especially for pharmaceutical products where carryover of metal impurities may cause serious problems in the production of many formulations ${ }^{2}$.

In order to address these problems, heterogeneous $\mathrm{Pd}$ catalysis is a promising option. $\mathrm{Pd}(0)$ or $\mathrm{Pd}(\mathrm{II})$ can be fixed to a solid support such as activated carbon (charcoal), zeolites and molecular sieves, metal oxides clays, alkali and alkaline earth salts, porous glass, organic polymers, or polymers embedded in porous glass. Different Pd nanoparticles are also active catalytic systems due to their large surface area ${ }^{7}$ and some of them have been also used for Hiyama cross-coupling reaction. Among heterogeneous Pd sources, palladium activated on carbon $(\mathrm{Pd} / \mathrm{C})$ is a commercially available, inexpensive and very stable under acid and basic conditions. Recently, different coupling procedures ${ }^{5}$ for carbon-carbon, carbonoxygen and carbon-nitrogen bond formation in the presence of $\mathrm{Pd} / \mathrm{C}$ have been developed. $\mathrm{Pd} / \mathrm{C}$-catalyzed Hiyama coupling reactions of aryl halides including ligand-free fashion 
were also reported $^{13 c}$, but they have limited scope with low availability of substrates or the use of highly polar organic solvents ${ }^{14}$.

We report herein, on the application of $\mathrm{Pd} / \mathrm{C}$ for the Hiyama cross-coupling of aryl halides with aryl siloxanes in the presence of $\mathrm{NaOH}$ as the base under aqueous conditions (Scheme-I).
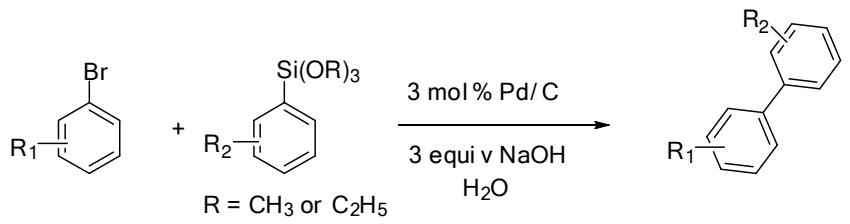

Scheme-I

\section{EXPERIMENTAL}

Experimental procedure for the Hiyama cross-coupling reaction: A mixture of aryl bromide $(1 \mathrm{mmol})$, aryl siloxane (1.2 mmol), $\mathrm{NaOH}(3 \mathrm{mmol}), \mathrm{Pd} / \mathrm{C}$ catalyst $(2 \mathrm{~mol} \%$ of $\mathrm{Pd})$ and distilled water $(3 \mathrm{~mL})$ was taken in a round-bottomed flask and stirred at $100{ }^{\circ} \mathrm{C}$ for appropriate time. After completion of the reaction (monitored by TLC) the catalyst was easily separated from the reaction mixture with simple filtration. After removing the solvent, the crude material was chromatographed on silica gel to afford the pure product. The spectroscopic data of all known compounds were identical to those reported in the literature.

\section{Spectroscopic data for the products}

Biphenyl (Table-2, entry 1): ${ }^{1} \mathrm{H}$ NMR $(300 \mathrm{MHz}$, $\left.\mathrm{CDCl}_{3}\right): \delta$ 7.67-7.32 (m, 2H), 7.36-7.42 (m, 4H), 7.52-7.56 $(\mathrm{m}, 4 \mathrm{H}) .{ }^{13} \mathrm{C} \mathrm{NMR}\left(75 \mathrm{MHz}, \mathrm{CDCl}_{3}\right): \delta 127.1,127.2,128.7$, 141.2. EI MS $(\mathrm{m} / \mathrm{z}): 154\left(\mathbf{M}^{+}\right)$.

4-Methoxy-biphenyl (Table-2, entry 2): ${ }^{1} \mathrm{H}$ NMR (300 $\left.\mathrm{MHz}, \mathrm{CDCl}_{3}\right): \delta 3.84$ (s, 3H), $6.92(\mathrm{~d}, 2 \mathrm{H}, J=8.9 \mathrm{~Hz}), 7.24-$ $7.27(\mathrm{~m}, 1 \mathrm{H}), 7.35-7.38(\mathrm{~m}, 2 \mathrm{H}), 7.47(\mathrm{~d}, 2 \mathrm{H}, J=8.9 \mathrm{~Hz})$, $7.50(\mathrm{~d}, 2 \mathrm{H}, J=7.9 \mathrm{~Hz}) .{ }^{13} \mathrm{C}$ NMR $\left(75 \mathrm{MHz}, \mathrm{CDCl}_{3}\right): \delta 55.3$, 114.2, 126.6, 126.7, 128.1, 128.6, 133.7, 140.8, 159.1. EI MS $(\mathrm{m} / \mathrm{z}): 184\left(\mathbf{M}^{+}\right)$.

2-Methoxy-biphenyl (Table-2, entry 3): ${ }^{1} \mathrm{H}$ NMR (300 $\left.\mathrm{MHz}, \mathrm{CDCl}_{3}\right): \delta 3.81(\mathrm{~s}, 3 \mathrm{H}), 6.93-7.00(\mathrm{~m}, 2 \mathrm{H}), 7.26-7.29$ $(\mathrm{m}, 3 \mathrm{H}), 7.34-7.38(\mathrm{~m}, 2 \mathrm{H}), 7.47(\mathrm{~d}, 2 \mathrm{H}, J=6.9 \mathrm{~Hz}) \cdot{ }^{13} \mathrm{C}$ NMR (75 MHz, $\mathrm{CDCl}_{3}$ ): $\delta 55.5,111.2,120.8,126.8,127.9$, 128.5, 129.5, 130.8, 138.5, 156.4. EI MS (m/z): $194\left(\mathrm{M}^{+}\right)$.

3-Methoxy-biphenyl (Table-2, entry 4): ${ }^{1} \mathrm{H}$ NMR (300 $\left.\mathrm{MHz}, \mathrm{CDCl}_{3}\right): \delta 386(\mathrm{~s}, 3 \mathrm{H}), 6.84(\mathrm{dd}, 1 \mathrm{H}, J=2.0 \mathrm{~Hz}, 8.1$ $\mathrm{Hz}), 7.07$ (s, 1H), $7.13(\mathrm{~d}, 1 \mathrm{H}, J=7.4 \mathrm{~Hz}), 7.31$ (t, 2H, $J=7.4$ $\mathrm{Hz}), 7.40(\mathrm{t}, 2 \mathrm{H}, J=8.1 \mathrm{~Hz}), 7.55(\mathrm{~d}, 2 \mathrm{H}, J=6.8 \mathrm{~Hz}) .{ }^{13} \mathrm{C}$ NMR (75 MHz, $\left.\mathrm{CDCl}_{3}\right): \delta 55.3,112.6,112.8,119.6,127.1$, $127.4,128.7,129.7,141.1,142.7,159.9$. EI MS (m/z): 184 $\left(\mathrm{M}^{+}\right)$.

4-Methyl-biphenyl (Table-2, entry 6): ${ }^{1} \mathrm{H}$ NMR (300 $\left.\mathrm{MHz}, \mathrm{CDCl}_{3}\right): \delta 2.50(\mathrm{~s}, 3 \mathrm{H}), 7.35(\mathrm{~d}, 2 \mathrm{H}, J=7.9 \mathrm{~Hz}), 7.42(\mathrm{t}$, $1 \mathrm{H}, J=7.6 \mathrm{~Hz}), 7.53(\mathrm{t}, 2 \mathrm{H}, J=7.6 \mathrm{~Hz}), 7.60(\mathrm{~d}, 2 \mathrm{H}, J=8.1$ $\mathrm{Hz}), 7.69$ (d, 2H, $J=7.2 \mathrm{~Hz}) .{ }^{13} \mathrm{C} \mathrm{NMR}\left(75 \mathrm{MHz}, \mathrm{CDCl}_{3}\right): \delta$ 21.1, 126.9, 127.0, 128.6, 129.4, 136.9, 138.3, 141.1. EI MS $(\mathrm{m} / \mathrm{z}): 168\left(\mathrm{M}^{+}\right)$.
2-Methyl-biphenyl (Table-2, entry 7): ${ }^{1} \mathrm{H}$ NMR (300 $\left.\mathrm{MHz}, \mathrm{CDCl}_{3}\right): \delta 2.26(\mathrm{~s}, 3 \mathrm{H}), 7.17-7.22(\mathrm{~m}, 4 \mathrm{H}), 7.26-7.31$ (m, 3H), 7.34-7.39 (m, 2H). ${ }^{13} \mathrm{C}$ NMR (75 MHz, $\left.\mathrm{CDCl}_{3}\right): \delta$ 20.4, 125.7, 126.7, 127.2, 128.0, 129.1, 129.7, 130.2, 135.3, 141.9. EI MS $(\mathrm{m} / \mathrm{z}): 168\left(\mathrm{M}^{+}\right)$.

4-Fluoro-biphenyl (Table-2, entry 9): ${ }^{1} \mathrm{H}$ NMR (300 $\left.\mathrm{MHz}, \mathrm{CDCl}_{3}\right): \delta$ 7.07-7.13 (m, 2H), 7.27-7.32 (m, 1H), 7.36$7.41(\mathrm{~m}, 2 \mathrm{H}), 7.47-7.53(\mathrm{~m}, 4 \mathrm{H}) .{ }^{13} \mathrm{C} \mathrm{NMR}\left(75 \mathrm{MHz}, \mathrm{CDCl}_{3}\right)$ : $\delta 115.4,115.7,126.9,127.2,128.6,128.8,137.3,140.2 . \mathrm{EI}$ $\operatorname{MS}(\mathrm{m} / \mathrm{z}): 172\left(\mathbf{M}^{+}\right)$.

1-Biphenyl-4-yl-ethanone (Table-2, entry 10): ${ }^{1} \mathrm{H}$ NMR $\left(300 \mathrm{MHz}, \mathrm{CDCl}_{3}\right): \delta 2.62$ (s, 3H), 7.34-7.38 (m, 1H), 7.41$7.45(\mathrm{~m}, 2 \mathrm{H}), 7.58(\mathrm{~d}, 2 \mathrm{H}, J=7.9 \mathrm{~Hz}), 7.65(\mathrm{~d}, 2 \mathrm{H}, J=8.9$ $\mathrm{Hz}), 8.00(\mathrm{~d}, 2 \mathrm{H}, J=8.9 \mathrm{~Hz}) \cdot{ }^{13} \mathrm{C} \mathrm{NMR}\left(75 \mathrm{MHz}, \mathrm{CDCl}_{3}\right): \delta$ 26.5, 127.1, 127.2, 128.2, 128.8, 128.9, 135.7, 139.8, 145.7, 197.6. EI MS $(\mathrm{m} / \mathrm{z}): 196\left(\mathrm{M}^{+}\right)$.

4-Nitro-biphenyl (Table-2, entry 11): ${ }^{1} \mathrm{H}$ NMR (300 $\left.\mathrm{MHz}, \mathrm{CDCl}_{3}\right): \delta 7.40-7.50(\mathrm{~m}, 3 \mathrm{H}), 7.59(\mathrm{~d}, 2 \mathrm{H}, J=8.3 \mathrm{~Hz})$, $7.72(\mathrm{~d}, 2 \mathrm{H}, J=8.3 \mathrm{~Hz}), 8.30(\mathrm{~d}, 2 \mathrm{H}, J=9.0 \mathrm{~Hz}) .{ }^{13} \mathrm{C} \mathrm{NMR}$ (75 MHz, $\left.\mathrm{CDCl}_{3}\right): \delta 124.0,127.3,127.7,128.8,129.1,138.7$, 147.6. EI MS $(\mathrm{m} / \mathrm{z}): 199\left(\mathbf{M}^{+}\right)$.

Biphenyl-4-carbaldehyde (Scheme 2): ${ }^{1} \mathrm{H}$ NMR (300 $\left.\mathrm{MHz}, \mathrm{CDCl}_{3}\right): \delta$ 7.37-7.49 (m, 3H), 7.58-7.62 (m, 2H), 7.72 $(\mathrm{d}, 2 \mathrm{H}, J=8.3 \mathrm{~Hz}), 7.93(\mathrm{~d}, 2 \mathrm{H}, \mathrm{J}=8.3 \mathrm{~Hz}) .{ }^{13} \mathrm{C} \mathrm{NMR}(75$ $\left.\mathrm{MHz}, \mathrm{CDCl}_{3}\right): \delta 127.3,127.6,128.4,128.9,130.2,130.7$, 135.1, 147.2, 191.9. EI MS $(\mathrm{m} / \mathrm{z}): 282\left(\mathrm{M}^{+}\right)$.

4,4',5,6-Tetramethoxybiphenyl-2-carbaldehyde (Scheme-II): IR (neat): 2940, 1683, 1587, 1464, 1324, 1141, 1087, $996 \mathrm{~cm}^{-1} .{ }^{1} \mathrm{H}$ NMR $\left(300 \mathrm{MHz}, \mathrm{CDCl}_{3}\right): \delta 3.56(\mathrm{~s}, 3 \mathrm{H})$, 3.86 (s, 3H), 3.96 (s, 3H), 3.97 (s, 3H), 6.93 (d, 2H, $J=8.3$ $\mathrm{Hz}), 7.22(\mathrm{~d}, 2 \mathrm{H}, J=9.0 \mathrm{~Hz}), 7.29(\mathrm{~s}, 1 \mathrm{H}), 9.63(\mathrm{~s}, 1 \mathrm{H}) .{ }^{13} \mathrm{C}$ $\mathrm{NMR}\left(75 \mathrm{MHz}, \mathrm{CDCl}_{3}\right): \delta 55.1,55.9,60.8,60.9,105.0,113.3$, 124.6, 129.7, 132.1, 134.1, 151.1, 152.7, 159.2, 191.3. ESI MS $(\mathrm{m} / \mathrm{z}): 303(\mathrm{M}+\mathrm{H})$.

4,4'-Dimethyl-[1,1';4',1'] terphenyl (Scheme-III): ${ }^{1} \mathrm{H}$ NMR (300 MHz, $\left.\mathrm{CDCl}_{3}\right): \delta 2.35(\mathrm{~s}, 6 \mathrm{H}), 7.19-7.26(\mathrm{~m}, 8 \mathrm{H})$, $7.33(\mathrm{~s}, 4 \mathrm{H}) .{ }^{13} \mathrm{C}$ NMR $\left(75 \mathrm{MHz}, \mathrm{CDCl}_{3}\right): \delta 20.5,125.7,127.2$, 128.8, 129.8, 130.3, 135.4, 140.3, 141.6. EI MS ( $\mathrm{m} / \mathrm{z}): 258\left(\mathrm{M}^{+}\right)$.

Tetraphenyl (Scheme-III): ${ }^{1} \mathrm{H}$ NMR $\left(300 \mathrm{MHz}, \mathrm{CDCl}_{3}\right)$ : $\delta 2.47(\mathrm{~s}, 9 \mathrm{H}), 7.24-7.48(\mathrm{~m}, 6 \mathrm{H}), 7.55-7.75(\mathrm{~m}, 6 \mathrm{H}), 7.82(\mathrm{~s}$, $3 \mathrm{H}) .{ }^{13} \mathrm{C} \mathrm{NMR}\left(75 \mathrm{MHz}, \mathrm{CDCl}_{3}\right): \delta 21.1,124.5,127.1,129.5$, 137.2, 138.3, 142.1. EI MS $(\mathrm{m} / \mathrm{z}): 348\left(\mathrm{M}^{+}\right)$.

4'-Methoxy-4-methyl-biphenyl (Table-3, entry 2): ${ }^{1} \mathrm{H}$ NMR (300 MHz, $\left.\mathrm{CDCl}_{3}\right): \delta 2.38(\mathrm{~s}, 3 \mathrm{H}), 3.82(\mathrm{~s}, 3 \mathrm{H}), 6.90(\mathrm{~d}$, $2 \mathrm{H}, J=9.0 \mathrm{~Hz}), 7.16(\mathrm{~d}, 2 \mathrm{H}, J=8.3 \mathrm{~Hz}), 7.38(\mathrm{~d}, 2 \mathrm{H}, J=8.3$ $\mathrm{Hz}), 7.44(\mathrm{~d}, 2 \mathrm{H}, J=9.0 \mathrm{~Hz}) \cdot{ }^{13} \mathrm{C} \mathrm{NMR}\left(75 \mathrm{MHz}, \mathrm{CDCl}_{3}\right): \delta$ 21.0, 55.3, 114.1, 126.5, 127.9, 129.4, 133.7, 136.3, 137.9, 158.8. EI MS $(\mathrm{m} / \mathrm{z}): 198\left(\mathrm{M}^{+}\right)$.

2,4'-Dimethyl-biphenyl (Table-3, entry 3): ${ }^{1} \mathrm{H}$ NMR (300 MHz, $\left.\mathrm{CDCl}_{3}\right): \delta 2.25(\mathrm{~s}, 3 \mathrm{H}), 2.41$ (s, 3H), 7.14-7.72 (m, $8 \mathrm{H}) .{ }^{13} \mathrm{C} \mathrm{NMR}\left(75 \mathrm{MHz}, \mathrm{CDCl}_{3}\right): \delta 20.5,21.2,125.7,127.0$, 128.7, 129.0, 129.8, 130.2, 136.3, 138.9. EI MS ( $\mathrm{m} / \mathrm{z}): 182\left(\mathrm{M}^{+}\right)$.

4'-Methoxy-4-methyl-biphenyl (Table-3, entry 4): ${ }^{1} \mathrm{H}$ NMR (300 MHz, $\left.\mathrm{CDCl}_{3}\right): \delta 2.35(\mathrm{~s}, 3 \mathrm{H}), 3.78(\mathrm{~s}, 3 \mathrm{H}), 6.88-$ 6.99 (m, 2H), 7.22-7.28 (m, 2H), 7.15 (d, 2H, $J=8.0 \mathrm{~Hz})$, $7.35(\mathrm{~d}, 2 \mathrm{H}, J=8.0 \mathrm{~Hz}) .{ }^{13} \mathrm{C} \mathrm{NMR}\left(75 \mathrm{MHz}, \mathrm{CDCl}_{3}\right): \delta 21.1$, 55.4, 111.1, 120.7, 128.3, 128.6, 129.3, 130.7, 131.4, 131.5, 136.4, 156.4. EI MS (m/z): $198\left(\mathrm{M}^{+}\right)$. 


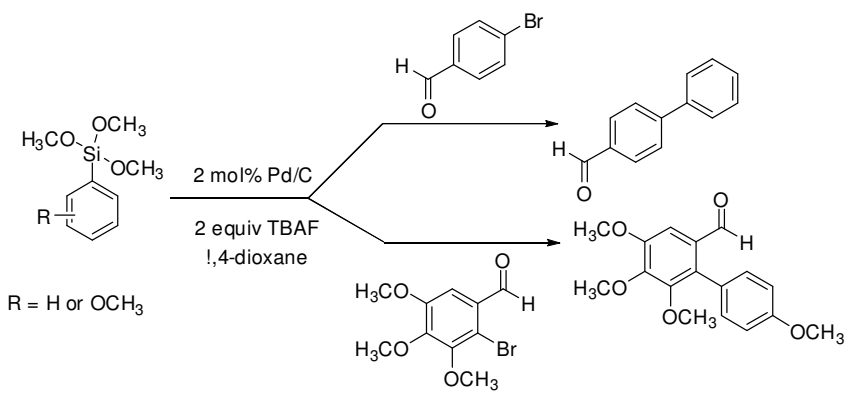

Scheme-II

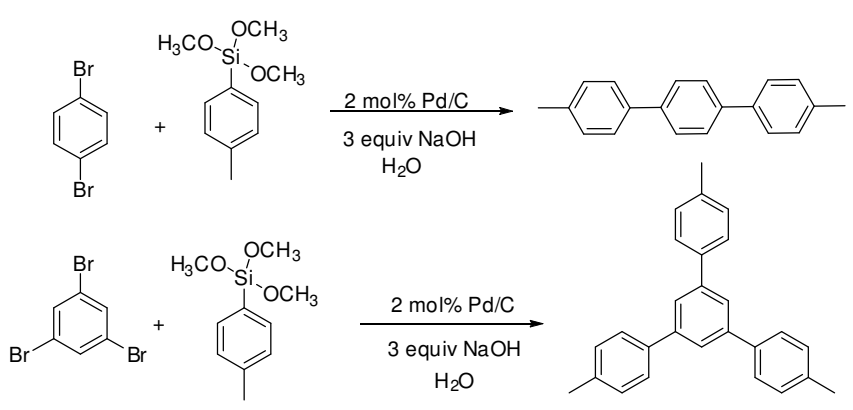

Scheme-III

\section{RESULTS AND DISCUSSION}

In an effort to develop a better catalytic system, initially we investigated the coupling of 4-bromotoluene with trimethoxyphenylsilane in pure water at $100{ }^{\circ} \mathrm{C}$ under open air by using different bases and the results are summarized in Table-1. The bases used in this study includes $\mathrm{NaOH}, \mathrm{KOH}$, $\mathrm{LiOH}, \mathrm{K}_{2} \mathrm{CO}_{3}, \mathrm{~K}_{3} \mathrm{PO}_{4}, \mathrm{Cs}_{2} \mathrm{CO}_{3}, \mathrm{KF}, \mathrm{NaHCO}_{3}$ and $\mathrm{NaOAc}$. The base has a pronounced effect in this reaction and out of which $\mathrm{NaOH}$ has been proven to the best base and $\mathrm{KOH}$ proven to be equally good as $\mathrm{NaOH}$ (Table-1, entries 1-2). $\mathrm{LiOH}, \mathrm{K}_{2} \mathrm{CO}_{3}$, $\mathrm{Cs}_{2} \mathrm{CO}_{3}$ and $\mathrm{KF}$ gave the product in low to moderate yields (Table-1, entries 3-6) and no product was formed with $\mathrm{K}_{3} \mathrm{PO}_{4}$, $\mathrm{NaHCO}_{3}$ and $\mathrm{NaOAc}$ (Table-1, entries 7). The control reaction conducted under identical conditions and devoid of $\mathrm{Pd} / \mathrm{C}$ gave no coupled product, despite a prolonged reaction time.

TABLE-1

SCREENING OF VARIOUS BASES FOR THE HIYAMA CROSSCOUPLING REACTION BETWEEN 4-BROMOTOLUENE AND TRIMETHOXYPHENYLSILANE

\begin{tabular}{ccc}
\hline Entry & Base & Yield (\%) \\
\hline 1 & $\mathrm{NaOH}$ & 80 \\
2 & $\mathrm{KOH}$ & 72 \\
3 & $\mathrm{LiOH}$ & 34 \\
4 & $\mathrm{~K}_{2} \mathrm{CO}_{3}$ & 16 \\
5 & $\mathrm{Cs}_{2} \mathrm{CO}_{3}$ & 14 \\
6 & $\mathrm{KF}$ & 41 \\
\hline
\end{tabular}

${ }^{a}$ Reaction conditions: 4-bromotoluene (1 mmol), trimethoxyphenylsilane $(1.2 \mathrm{mmol}), \mathrm{Pd} / \mathrm{C}(2 \mathrm{~mol} \%), \mathrm{NaOH}(3$ equiv), water $(3 \mathrm{~mL})$ stirred at $100{ }^{\circ} \mathrm{C}$ for $6 \mathrm{~h}$.

We observed that the best conditions developed for trimethoxyphenylsilane and 4-bromotoluene were found to be broadly applicable. When they were applied to a variety of neutral, electron-rich and electron-poor aryl halides to investigate the synthetic scope of the reaction, the corresponding biaryls were isolated in good to excellent yields. Our preparative

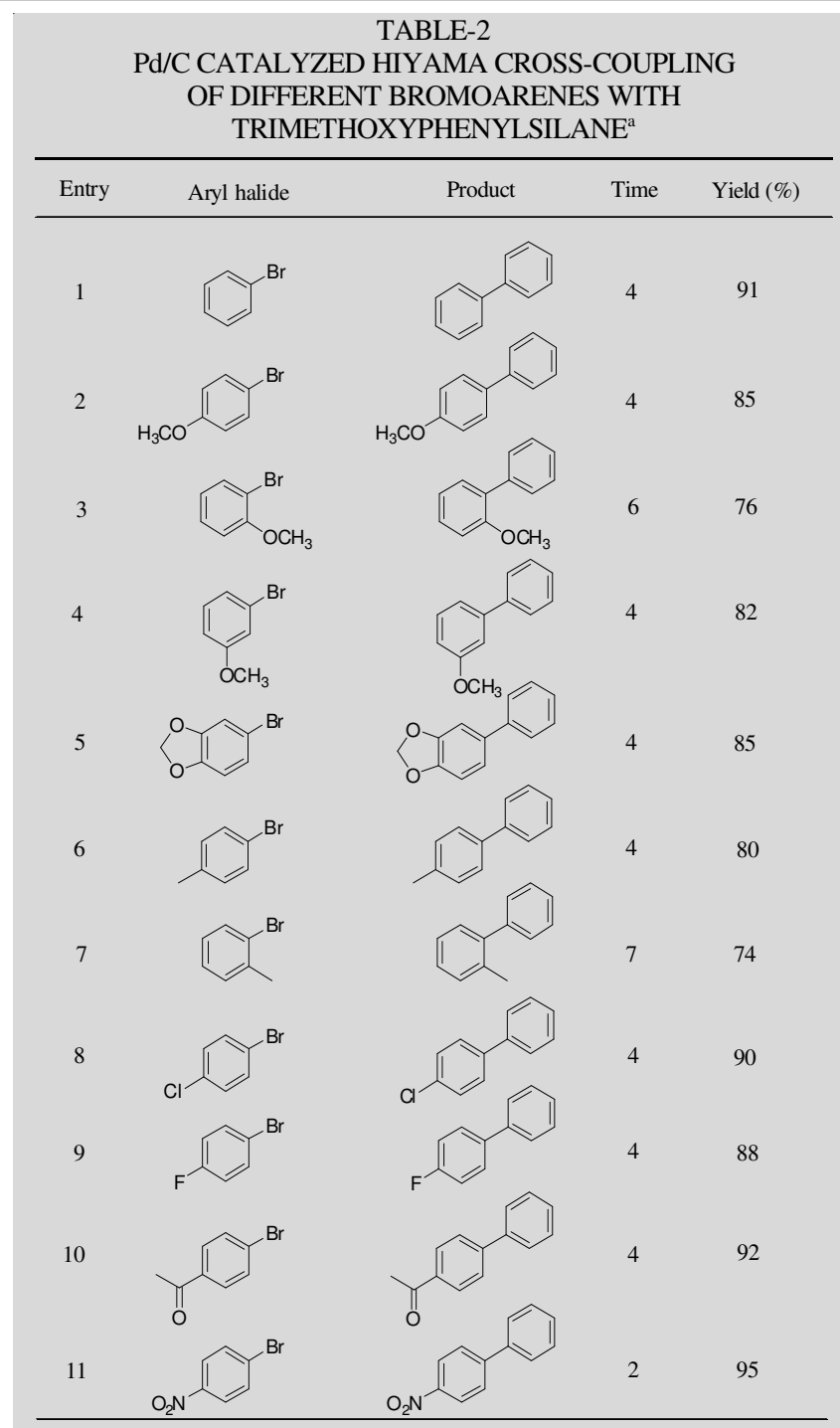

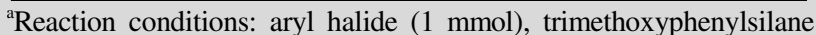
(1.2 mmol), $2 \mathrm{~mol} \%$ of $\mathrm{Pd} / \mathrm{C}, \mathrm{NaOH}$ (3 equiv) water ( $3 \mathrm{~mL})$ stirred at $100^{\circ} \mathrm{C}$

results are presented in Table-3. Unsubstituted as well as 4-methyl, 4-methoxy, 3-methoxy, 3,4-methylenedioxide substituted bromobenzenes underwent smooth reaction with good yields when compared to 2-methyl and 2-methoxy bromobenzenes (Table-3, entries 1-7). Furthermore, the reaction was extended to other aryl halides having electron withdrawing groups to generate biaryl products in good to excellent yields (Table-3, entries 8-11).

Unfortunately, this catalytic system was ineffective for the reaction with the aldehyde substituted bromo arenes under the optimized reaction conditions (Scheme-II). However, best results were obtained by changing the solvent from $\mathrm{H}_{2} \mathrm{O}$ to 1,4-dioxane and $\mathrm{NaOH}$ to TBAF. Under the above conditions the $\mathrm{Pd} / \mathrm{C}$ possessed good catalytic activity for both 4-bromo benzaldehyde and 2-bromo-3,4,5-trimethoxy benzaldehydes. The formed product 4,4',5,6-tetramethoxybiphenyl-2carbaldehyde is useful for the synthesis of antitumor agent allocolchicine methyl ether.

To show the convenience of our approach for the synthesis of multivalent structures, the coupling of 1,2-dibromobenzene and 1,3,5-tribromobenzenes with $p$-methyl phenyltrimethoxysilane 


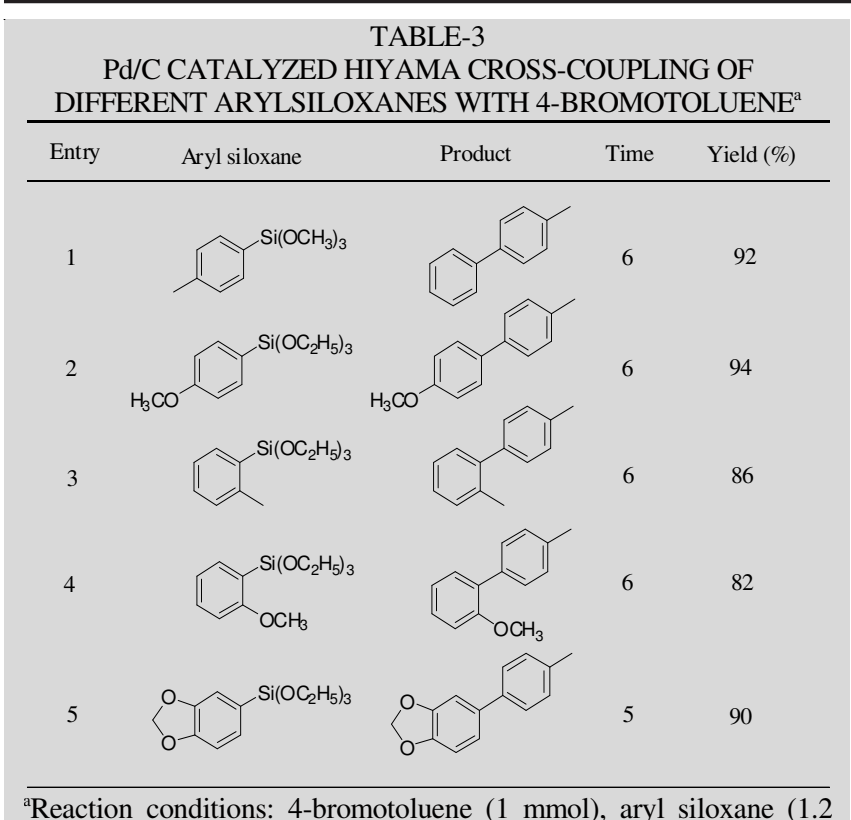

$\mathrm{mmol}), 2 \mathrm{~mol} \%$ of $\mathrm{Pd} / \mathrm{C}, \mathrm{NaOH}$ (3 equiv) water $(3 \mathrm{~mL})$ stirred at $100{ }^{\circ} \mathrm{C}$.

was carried out under the optimized reaction conditions to afford high yields of terphenyl and tetraphenyl respectively (Scheme-III).

Given the remarkably high levels of catalytic activity displayed with $\mathrm{Pd} / \mathrm{C}$ catalyst, we investigated its use for the preparation of biaryls from 4-bromotoulene and different phenyl trimethoxysilanes. As can be seen from the Table-3, phenyl triethoxysilanes with electron-donating groups such as methoxy, methyl and 3,4-methylenedioxy groups (Table-3, entries 1,2 and 5) underwent smooth reaction with excellent yields compared to those with electron-donating groups (Table3 , entries 3 and 4) present in the ortho-position of the phenyl ring.

\section{Conclusion}

In conclusion, we have developed a simple and efficient method for the synthesis of biaryls via fluoride-free aqueous Hiyama cross-coupling of bromoarenes with aryl siloxanes using heterogeneous $\mathrm{Pd} / \mathrm{C}$ as the catalyst under mild reaction conditions. This protocol can be used to generate a diverse range of biaryls in good to excellent yields.

\section{REFERENCES}

1. (a) D.A. Horton, G.T. Bourne and M.L. Smythe, Chem. Rev., 103, 893 (2003).; (b) G. Bringmann, C. Gunther, M. Ochse, O. Schupp and S. Tasler, in eds: W. Herz, H. Falk, G.W. Kirby and R.E. Moore, Biaryls in Nature: A Multi-Facetted Class of Stereochemically, Biosynthetically, and Pharmacologically Intriguing Secondary Metabolites In: Progress in the Chemistry of Organic Natural Products, Springer-Verlag: New York, Volume 82 (2001); (c) P.J. Hajduk, M. Bures, J. Praestgaard and S.W. Fesik, J. Med. Chem., 43, 3443 (2000); (d) G.W. Bemis and M.A. Murcko, J. Med. Chem., 39, 2887 (1996).

2. (a) A. Markham and K.L. Goa, Drugs, 54, 299 (1997); (b) K.F. Croom and G.M. Keating, Am. J. Cardiovasc. Drugs, 4, 395 (2004); (c) M. Sharpe, B. Jarvis and K.L. Goa, Drugs, 61, 1501 (2001); (d) S. Yusuf, Am. J. Cardiol., 89, 18 (2002).

3. M.E. Matheron and M. Porchas, Plant Dis., 88, 665 (2004).

4. (a) U. Schmidt, V. Leitenberger, H. Griesser, J. Schmidt and R. Meyer, Synthesis, 1248 (1992); (b) U. Schmidt, R. Meyer, V. Leitenberger, H. Griesser and A. Lieberknecht, Synthesis, 1025 (1992).

5. E. Poetsch, Kontakte, 2, 15 (1988).

6. (a) N. Miyaura, K. Yamada and A. Suzuki, Tetrahedron Lett., 20, 3437 (1979); (b) S.P. Stanforth, Tetrahedron, 54, 263 (1998); (c) A. Suzuki, Pure Appl. Chem., 63, 419 (1991); (d) J.P. Wolfe and S.P. Buchwald, Angew. Chem. Int. Ed., 38, 2413 (1999); (e) A. Zapf and M. Beller, Chem. Eur. J., 6, 1830 (2000); (f) R.B. Bedford, C.S.J. Cazin, S.J. Coles, T. Gelbrich, P.N. Horton, M.B. Hursthouse and M.E. Light, Organometallics, 22, 987 (2003).

7. (a) S. Baba and E. Negishi, J. Am. Chem. Soc., 98, 6729 (1976); (b) C. Dai and C. Fu, J. Am. Chem. Soc., 123, 2719 (2001).

8. (a) K. Tamao, K. Sumitani and M. Kumada, J. Am. Chem. Soc., 94, 4374 (1972); (b) W.A. Herrmann, V.P.W. Bohm and C. Reisinger, J. Organomet. Chem., 576, 23 (1999).

9. (a) Y. Hatanaka and T. Hiyama, J. Org. Chem., 53, 918 (1988); (b) K. Gouda, E. Hagiwara, Y. Hatanaka and T. Hiyama, J. Org. Chem., 61, 7232 (1996); (c) M.E. Mowery and P. DeShong, Org. Lett., 1, 2137 (1999); (d) J. Lee and G.C. Fu, J. Am. Chem. Soc., 125, 5616 (2003); (e) S. Riggleman and P. Deshong, J. Org. Chem., 68, 8106 (2003); (f) H.M. Lee and S.P. Nolan, Org. Lett., 2, 2053 (2000).

10. (a) J.K. Stille, Pure Appl. Chem., 57, 1771 (1985); (b) J.K. Stille, Angew. Chem. Int. Ed., 25, 508 (1986); (c) V. Farina, V. Krishnamurthy and W. Scott, J. Org. React., 50, 1 (1997); (d) J. Hassan, M. Sévignon, C. Gozzi, E. Schulz and M. Lemaire, Chem. Rev., 102, 1359 (2002); (e) A.F. Littke and G.C. Fu, Angew. Chem. Int. Ed., 41, 4176 (2002).

11. (a) E. Hagiwara, K. Gouda, Y. Hatanaka and T. Hiyama, Tetrahedron Lett., 38, 439 (1997); (b) M. Murata, R. Shimazaki, S. Watanabe and Y. Masuda, Synthesis, 2231 (2001).

12. (a) C. Wolf and R. Lerebours, Org. Lett., 6, 1147 (2004); (b) Á. Gordillo, E. de Jesús and C. López-Mardomingo, Org. Lett., 8, 3517 (2006).

13. (a) S. Shi and Y. Zhang, J. Org. Chem., 72, 5927 (2007); (b) B.C. Ranu, R. Dey and K. Chattopadhyay, Tetrahedron Lett., 49, 3430 (2008); (c) T. Huang and C.J. Li, Tetrahedron Lett., 43, 403 (2002); (d) D. Srimani, S. Sawoo and A. Sarkar, Org. Lett., 9, 3639 (2007); (e) E. Alacid and C. Najera, Adv. Synth. Catal., 348, 945 (2006); (f) E. Alacid and C. Najera, Adv. Synth. Catal., 348, 2085 (2006).

14. A. Komaromi, F. Szabo and Z. Novak, Tetrahedron Lett., 51, 5411 (2010). 